\title{
Saúde, medicamentos, marketing e médicos
}

Luís dos Santos Ferreira*

\section{RESUMO}

O presente artigo introduz uma visão muito sintética da situação da Saúde em Portugal e do seu posicionamento em relação a outros países com um grau de desenvolvimento semelhante. Descreve as iniquidades ainda existentes e em especial o facto de os gastos com a saúde apresentarem um peso muito significativo em relação ao PIB, desproporcionado em relação à amplitude e à qualidade dos mesmos. Especifica o processo de prestação de cuidados de saúde e o papel fulcral do médico e do medicamento.

Após uma breve caracterização da indústria farmacêutica procura-se escalpelizar a relação entre o marketing farmacêutico e o médico enquanto prescritor. Da análise da situação o autor ressalta dois factos: a importância da informação na prescrição médica e a profunda assimetria existente entre a informação veiculada pela indústria farmacêutica e a informação independente. Desta assimetria ressalta a dependência da intensidade de prescrição de uma dada marca de medicamento relativamente à intensidade da informação veiculada pela indústria farmacêutica, junto do prescritor.

Neste texto são, ainda, analisadas as formas e meios usados pela indústria para promover os produtos, o seu peso na estrutura de custos das empresas, e são indicadas práticas impróprias e medidas que procuram limitar os seus efeitos negativos.

Palavras-Chave: Medicamentos; Publicidade; Médicos; Custo em Cuidados de Saúde.

\section{INTRODUÇÃO}

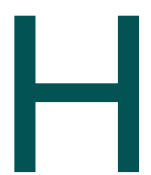

á cerca de sessenta anos (1948), de acordo com o artigo 57 da Carta das Nações Unidas, foi fundada a OMS (Organização Mundial de Saúde), uma agência da ONU (Organização das Nações Unidas) especializada na promoção da saúde, tendo como objectivo o fomento do mais elevado estado de saúde possível para todos os povos. Na Constituição e Objectivos da OMS é definido que a saúde é um estado de completo bem-estar físico, mental e social e não só um estado de ausência de doença ou enfermidade. A $13^{\text {a }}$ Assembleia da OMS decidiu que os principais objectivos dos Governos e da OMS nas décadas futuras seriam «que os cidadãos de todo o mundo atingissem no ano 2000 um nível de saúde que lhes permitisse levar uma vida social e economicamente produtiva». ${ }^{1}$

Em 2007, a definição inicial de Saúde da OMS foi complementada com a boa capacidade de o indivíduo se inserir social e economicamente, o que possibilita definir indicadores operacionais que permitam uma

*Afiliação profissional: Consultor de Gestão avaliação mais adequada dos progressos verificados, no sentido de concretizar o objectivo de «Saúde para todos». ${ }^{2}$ Para atingir esse objectivo são necessários meios de que todos os cidadãos possam usufruir para manter ou recuperar o seu estado de saúde. Esses meios compreendem um Sistema de Saúde equitativo e uma estrutura fornecedora de cuidados de saúde a que todos tenham direito, por igual.

\section{O SISTEMA DE SAÚDE PORTUGUÊS}

O sistema de saúde português adoptou como objectivos base os da Organização Mundial de Saúde. Em Portugal, a saúde é um direito constitucionalmente garantido tendencialmente gratuito. Assenta basicamente em estruturas estatais, tanto a nível de cuidados de saúde primários como secundários, consagrando o princípio de que os serviços sejam disponibilizados e estruturados no interesse do utente. Este sistema é complementado por sistemas de saúde comparticipados e privados. Os sistemas privados nos últimos anos têm assumido um peso relativo cada vez maior. O sistema português é, nos seus princípios teóricos, muito favorável aos utentes, mas os resultados e a prática não têm 
mostrado o que se esperaria das boas intenções do legislador.

O estado da Saúde em Portugal corresponde ao estado de desenvolvimento do país. Apresenta um conjunto de assimetrias significativas e, desde 2001, a situação de estagnação no desenvolvimento económico e social tem influenciado as medidas no campo da saúde. A situação económica em Portugal está marcada por um deficit crónico do orçamento que sucessivos governos têm tentado colmatar, diminuindo o investimento público e as despesas correntes e tentando aumentar as receitas. Sendo as despesas com a saúde a segunda maior rubrica inscrita no orçamento do Estado (a primeira é a Educação), os sucessivos Governos têm tentado limitar estes custos diminuindo as despesas correntes e o investimento, nomeadamente através da contenção das verbas destinadas à aquisição de bens e serviços, as despesas de pessoal e os investimentos, ao mesmo tempo que aumentam a participação dos utentes nos custos de saúde, generalizando e aumentando as chamadas taxas moderadoras.

\section{A SITUAÇÃO DA SAÚDE EM PORTUGAL FACE AO RESTO DO MUNDO}

Os estudos publicados no World Health Report (OMS) até 2006 comprovam a noção de que Portugal se encontra longe de atingir a concretização dos objectivos definidos na Carta da OMS. Em comparação com 192 países que fazem parte da OMS, Portugal situa-se em $27^{\circ}$ em termos de desenvolvimento humano (FNUAD, 2000) e em $30^{\circ}$, quando é avaliado o estado de saúde no país (OMS, 2006), sendo o último dos países da Zona Euro. Isto, apesar dos ganhos em saúde obtidos por Portugal nas últimas três décadas, consequência directa da criação do Serviço Nacional de Saúde.

No Sistema de Saúde português verifica-se existir alguma iniquidade e, paradoxalmente, os custos dos cuidados de saúde são elevados. Existem subsistemas de saúde que conferem aos beneficiários mais cuidados de saúde, mais hipóteses de escolha e de reembolsos mais generosos que os do SNS. Compreende-se, assim, que a existência em simultâneo do acesso ao Serviço Nacional de Saúde e a subsistemas com uma filosofia de acção divergente dê origem a situações de diferenciação no tratamento dos cidadãos consoante o subsistema em que estão inseridos e a sua capacidade econó- mica. Verifica-se, ainda, que em Portugal existe maior propensão ao consumo de cuidados de saúde pelas famílias com rendimentos mais elevados.

Nos últimos tempos tem-se assistido ao aumento do número e dimensão de unidades privadas de prestação de cuidados de saúde associadas a empresas especializadas e a seguros de saúde. Os grandes grupos económico-financeiros da banca e seguros, como por exemplo o grupo BES, o grupo Mello e mesmo a Caixa Geral de Depósitos, têm aumentado, de forma muito expressiva, a sua presença no sector da Saúde. Tudo leva a crer que essas entidades com fins lucrativos estão a captar uma parte cada vez maior dos cuidados de saúde através de esquemas de seguros de saúde. Por outro lado, assistiu-se a uma tentativa de racionalização das estruturas do SNS, que suscitou muitas reacções negativas por parte dos utentes e agentes da saúde (médicos, enfermeiros, outros profissionais de saúde). Essa reestruturação que visava a redução dos custos dos Serviços de Saúde comparticipados pelo Estado está, ao que parece, em «banho-maria», estando a ser implantadas medidas pontuais.

O sub-financiamento crónico do SNS é um limite à prossecução do objectivo da igualdade do acesso à saúde, colocando os mais pobres e, muitas vezes, mais necessitados em condição de desigualdade. O doente cada vez paga mais pelos cuidados de saúde que recebe, não existindo uma protecção suficiente dos mais doentes e dos mais pobres. Apesar disso, há que reconhecer que se verificaram, na última década, ganhos pontuais em problemas específicos de saúde, tais como no tratamento e prevenção da SIDA, tuberculose, diabetes, asma e cancro, entre outras.

No entanto, quando se compara Portugal com outros países da OCDE, a despesa na saúde peca pela utilização de um elevado nível de recursos em relação ao PIB, uma despesa excessiva com fármacos, assim como uma maior despesa com o sector privado, em relação a outros países com Serviço Nacional de Saúde (SNS). ${ }^{3} \mathrm{O}$ peso das verbas orçamentais em saúde representa cerca de $12 \%$ da despesa efectiva do Estado e $6 \%$ do PIB, sendo que mais de $2 \%$ do PIB é gasto em medicamentos e meios de diagnóstico privados.

O desempenho em relação à equidade, eficiência, responsabilização e capacidade de resposta do Sistema Público de Saúde fica aquém do que seria de esperar, 
tendo em conta o peso das Despesas Orçamentais. Gastamos demais para o nível de cuidados de saúde de que usufruímos. As causas para esta situação são de natureza organizativa. As formas organizacionais estão desactualizadas e as tentativas de reforma do sistema de saúde nunca foram completamente implementadas, conduzindo a situações de insuficiência e desigualdade no que respeita à prestação dos cuidados de saúde. ${ }^{4}$

\section{OS MEDICAMENTOS EM PORTUGAL}

No processo de prestação de cuidados de saúde, seja qual for o sistema de cuidados de saúde a que um indivíduo recorre, quando em situação de alteração da normalidade do seu estado de saúde, o médico, apoiado por outros técnicos de Saúde e meios auxiliares, procura efectuar um diagnóstico. Este diagnóstico leva à definição de um tratamento que, supostamente, irá contribuir para repor ou melhorar o estado de saúde do indivíduo. Em grande parte dos casos o tratamento inclui a utilização de medicamentos, recomendados sob a forma de uma receita médica, sendo praticamente todos os actos médicos efectuados mediante utilização de medicamentos.

Segundo o Dec.-Lei 71/91, de 8 de Fevereiro, medicamento é «toda a substância ou associação de substâncias que possua propriedades curativas ou preventivas de doenças e dos seus sintomas, do homem ou do animal, com vista a estabelecer um diagnóstico médico ou a restaurar, corrigir ou modificar as suas funções (fisiológicas)». ${ }^{5}$

A produção de medicamentos está fortemente legislada e constitui um sector económico privado, com fins lucrativos. Os medicamentos são produzidos ou importados por empresas especializadas, os laboratórios e importadores farmacêuticos, distribuídos pelos grossistas às farmácias; estas vendem-nos directamente ao consumidor final. O sistema hospitalar é abastecido directamente pelos laboratórios e importadores devidamente autorizados.

No total, em 2007, estimava-se que o mercado de medicamentos vendidos através das farmácias representava para os Laboratórios e importadores um valor próximo de 2.600.000.000 Euros (dois mil e seiscentos milhões de euros), enquanto o Mercado Hospitalar (Público e Privado) representava cerca de 600 a $700 \mathrm{mi}$ lhões de euros. No total, os Medicamentos representam cerca de 2,2\% do PIB português, de acordo com dados publicados pela IMS Health, citada por Pedro Rebelo no seu trabalho de Mestrado e pelos números do INE sobre o PIB, em 2007.

Ainda segundo a IMS Health, cerca de $21 \%$ do total de medicamentos vendidos em Portugal foi fornecido aos Hospitais e $79 \%$ foi vendido através das Farmácias. ${ }^{6}$ $\mathrm{O}$ consumo de medicamentos, quer em valor, quer em unidades, tem crescido regularmente. Desde o princípio do século o mercado farmacêutico cresceu, em média, $6,9 \%$ ao ano, muito acima da inflação no período medido pelo Índice de Potencial de Consumo (IPC). ${ }^{6}$

Em unidades, venderam-se em 2007 cerca de 275 milhões de unidades (Health IMS, fonte citada). ${ }^{6}$ De notar que o crescimento do mercado em unidades, ainda segundo a fonte anteriormente citada, tem sido muito inferior ao crescimento em valores. Em média, desde 2000 até 2007, o crescimento em valor situa-se próximo dos $6,9 \%$ enquanto em unidades não ultrapassa os $2,6 \%$. Cerca de $13 \%$ a $14 \%$ do valor corresponde a genéricos, quota que, comparada com outros países da U.E., é muito pequena. Em muitos países da Europa, a quota dos genéricos, em valor, está acima dos $30 \%$ do mercado e tem vindo a crescer. ${ }^{7}$

Estudos feitos em 1998 para a Food and Drug Administration revelaram que nos Estados Unidos da América e no Canadá o mercado de medicamentos genéricos atingiu um volume de $40 \% .{ }^{8}$ Estimativas publicadas nesse estudo apontam para uma poupança de cerca de 10 biliões de dólares nos E.U.A. e de 1 bilião de dólares no Canadá devido ao maior uso de genéricos. ${ }^{8}$

Estudos mais recentes indicam que o mercado de genéricos em países como o Reino Unido e a Dinamarca atingem já quotas de $49 \%$ e $60 \%$, respectivamente. ${ }^{6}$ Por seu lado há países em que a popularidade dos medicamentos genéricos é reduzida, como em Espanha, França, Portugal e Itália. Tal diferença pode ser explicada pela diferente intervenção governamental, pelas intervenções directas no direito à propriedade industrial e pelas políticas de marketing seguidas pela Indústria Farmacêutica, ainda segundo a European Generics Association. ${ }^{7}$ Mais recentemente, a IMS prevê que mais de 2/3 de todas as prescrições nos EUA, em 2008, sejam de genéricos. ${ }^{6}$

O mercado do medicamento tem vindo a aumentar, com a evolução tecnológica e com o aumento do nível 
e esperança de vida. Vive-se mais e são necessários mais cuidados de saúde. A nível mundial prevê-se que o mercado dos medicamentos de prescrição aumente entre 5 a $6 \%$ em 2008, valor um pouco abaixo dos 6 a $7 \%$ obtidos em 2007, mas muito acima do crescimento económico mundial previsto pela OCDE para igual período. O volume de vendas previsto para 2008 a nível mundial situar-se-á entre US\$ 735 a 745 biliões. $^{6}$

Segundo o INFARMED, dos 54 fármacos mais vendidos em Portugal, apenas seis custam aos portugueses menos do que os mesmos vendidos em Espanha, França e Itália, países que servem de referência para estabelecer os preços em Portugal. Num dos casos, o de um genérico de que o INFARMED não menciona a molécula activa, o custo chega a ser $308 \%$ superior ao valor mais baixo praticado nestes três países. Estes 54 medicamentos representam $17 \%$ do mercado (em quantidades vendidas) e têm preços mais altos, em média, entre os 18 e os $40 \%$, comparados com os valores praticados em outros estados europeus. ${ }^{9}$

Em 2007, o então Ministro da Saúde, Prof. Doutor António Correia de Campos, na sua intervenção na conferência Indústria Farmacêutica:Desafios para a Inovação e Saúde, organizada pelo Diário Económico a $30 \mathrm{de}$ Maio de 2007, fez a afirmação seguinte «... os preços excessivos verificam-se principalmente nos medicamentos mais antigos. Os medicamentos que foram introduzidos no mercado antes de 1990 são mais caros entre 175 e $35 \%$. A situação é ainda mais grave no caso dos fármacos introduzidos até 2000, em que «a média da diferença é $34 \%$ mas com valores desviantes, entre os $239 \%$ e os $130 \%$ ». A conclusão é óbvia: gastamos demais em Medicamentos. ${ }^{10}$

\section{UM MERCADO MUITO INTERESSANTE}

Para aproveitar o potencial de mercado de qualquer medicamento, a indústria farmacêutica despende grandes somas para influenciar a sua prescrição, actuando através de todos os meios possíveis sobre os prescritores, essencialmente os Médicos.

Estudos efectuados pela OCDE e investigadores independentes mostram que a indústria farmacêutica, nos principais países da Europa e nos EUA, gasta entre $20 \%$ e $27 \%$ dos seus proveitos em publicidade e vendas, sendo o sector com maiores gastos promocionais, seguido pela indústria da perfumaria e cosmética (15\%).

\begin{tabular}{|lr|}
\hline \multicolumn{2}{|l|}{ QUADRO I. Estrutura de custos da Indústria Farmacêutica } \\
\hline Produção & $25 \%$ \\
\hline Marketing & $24 \%$ \\
\hline I\&D & $13 \%$ \\
\hline Outros & $10 \%$ \\
\hline Lucro operacional & $28 \%$ \\
\hline Total & $100,00 \%$ \\
\hline
\end{tabular}

Embora estes estudos já tenham alguns anos, muitos indicadores apontam no sentido de que estas despesas têm aumentado. ${ }^{11-13}$

Jacobezone, no estudo da OCDE já citado e referido do CPB (Central Planbureau dos Países Baixos) aponta para a estrutura de custos da Indústria Farmacêutica em 1989 referida no Quadro I. ${ }^{11,14}$

De salientar que muitos autores são de opinião de que uma parte significativa das despesas de Investigação e Desenvolvimento (I\&D) são de facto despesas promocionais, como os ensaios comprovativos, que essencialmente visam criar o hábito de prescrição de uma determinada droga e, em muitos casos, são de ética científica duvidosa. Também é de salientar que análises, mais recentes, efectuadas na União Europeia (U.E.), por exemplo no National Health Service do Reino Unido, em amostras significativas de dados referentes aos gastos com promoção e venda na IF, apontam para o seu aumento.

\section{COMO SE EXPLICA ESTA INTENSIDADE DAS ACÇÕES DE MARKETING?}

O mercado dos medicamentos é de facto um mercado muito especial. É altamente complexo, com uma enorme variedade de intervenientes, desde as empresas farmacêuticas, aos distribuidores, farmácias, médicos e outros técnicos de saúde, doentes, entidades prestadoras de cuidados de Saúde (privadas e públicas) e seguradoras. Em primeiro lugar é um mercado global, de oligopólios, utilizando de forma intensa a lei das patentes com base nas moléculas (patente produto). É um mercado em que a inovação desempenha um papel muito importante. Todas as grandes empresas farmacêuticas procuram inovar, introduzindo novas moléculas que são mais eficazes, supostamente, do que as 
que vão substituir. O mercado farmacêutico não é homogéneo, deve ser examinado de acordo com as diferentes terapêuticas, por exemplo, o mercado dos anti-inflamatórios, dos hipotensores, dos anti-ulcerosos, dos antidiabéticos, etc. É dentro destas classes terapêuticas que se estabelecem oligopólios; cada uma delas é dominada por uma a quatro moléculas diferentes que representam, em geral, mais de $80 \%$ do volume de vendas da classe considerada.

Os dados estatísticos mostram que, por classe terapêutica, os três produtos mais vendidos detêm, em geral, cerca de $80 \%$ das vendas dessa classe. Muitas vezes, uma classe terapêutica é dominada por uma ou duas moléculas, como é o caso da úlcera péptica. ${ }^{6}$

O mercado dos medicamentos é também um mercado fortemente regulamentado pelos Governos que intervêm na aprovação dos medicamentos a introduzir num dado país, na fixação do seu preço de venda ao público e, sempre que existe comparticipação do Estado, na compra do medicamento, na percentagem de comparticipação e na possibilidade da sua prescrição nos serviços de saúde públicos.

Se analisarmos objectivamente as diferentes regulamentações do mercado farmacêutico, verificamos que as diferentes barreiras, muitas vezes criadas para controlarem a actividade das empresas, contribuem para a manutenção e o desenvolvimento de oligopólios, garantindo exclusivos de exploração do mercado, com a concessão de autorização de entrada de medicamentos durante o período de duração da patente e, após esta expirar, com a concessão de direitos de entrada de moléculas iguais. Disso, é exemplo a passagem do regime de patentes com base em processos para o regime de patentes com base na molécula ou produto que eliminou grande parte da concorrência. No regime de processo era possível maior concorrência dado que um novo processo de produção permitia a introdução de produtos iguais aos primeiros inovadores, facilitando a concorrência pelo preço.

No mercado do medicamento existem, ainda, organismos oficiais que acompanham os medicamentos existentes no mercado, quer quanto à sua qualidade, quer quanto às práticas de marketing utilizadas pelas empresas do sector farmacêutico que fornecem informação sobre os medicamentos aos prescritores e utilizadores. Em Portugal, essa função está cometida ao
INFARMED. No entanto, a maioria da informação sobre os produtos farmacêuticos tem origem nas próprias empresas farmacêuticas e não em organismos independentes. A informação é assimétrica. Os meios informativos utilizados pelas entidades independentes são de um volume e intensidade muitíssimo inferiores aos que a indústria farmacêutica aplica em publicidade, promoção e vendas.

Actualmente, quando aparece uma das raras inovações terapêuticas, é normal seguir-se o aparecimento de produtos com substâncias químicas similares (produtos «me too») que são substitutos próximos dos produtos inovadores, muitas vezes com acção terapêutica quase igual. Estes produtos também são patenteados e raramente concorrem pelo preço, procurando aumentar a sua quota de mercado em relação ao «leader», através de uma grande intensidade de promoção.

Mais ainda, a participação das entidades oficiais da saúde no preço dos medicamentos facilita a sua aquisição e uso, dado baixar o preço pago pelo utente, facilitando o aumento da procura.

Em termos de mercado, das classes terapêuticas listadas na CID (Classificação Internacional de Doenças), OMS, revisão de 1975, são perto de 100 as mais importantes. Fundamentalmente, nestas classes terapêuticas, competem a nível supra nacional cerca de 150 empresas, promovendo mais de 1.200 moléculas activas. Destas 150 empresas, 50 detêm mais de $80 \%$ das vendas a nível mundial, sendo que as dez mais importantes em Portugal são todas multinacionais, sendo uma única de capital português, a BIAL. Todas as outras são empresas de grande dimensão actuando, directa ou indirectamente, em todos os 194 países do Mundo.

Temos, assim, que o mercado farmacêutico difere de outros mercados em muitos aspectos, o que explica a enorme intensidade de promoção que o caracteriza, nomeadamente:

- É fortemente regulado, sendo muito limitada a introdução de produtos no mercado

- Tem um número muito heterogéneo de intervenientes

- É um mercado de prescrição onde o médico assume um papel relevante

- Envolve uma grande quantidade de informação assimétrica

- Não é um mercado homogéneo, encontra-se seg- 


QUADRO II. As 10 maiores empresas farmacêuticas operando em PORTUGAL -
Mercado Ambulatório
\begin{tabular}{c|l|c|c} 
Ordem & Empresa & Vendas estimadas em 2007 & Quota de Mercado \\
\hline 1 & Pfizer & $151.774 .923 €$ & $5,95 \%$ \\
\hline 2 & Merck Sharp\&Dome & $144.616 .045 €$ & $5,67 \%$ \\
\hline 3 & Servier & $99.645 .351 €$ & $3,91 \%$ \\
\hline 4 & Aventis-Sanofi & $96.989 .868 €$ & $3,80 \%$ \\
\hline 5 & Novartis & $91.721 .624 €$ & $3,60 \%$ \\
\hline 6 & Sanofi-BMS & $90.695 .365 €$ & $3,56 \%$ \\
\hline 7 & Bayer & $89.263 .461 €$ & $3,50 \%$ \\
\hline 8 & Astra Zeneca & $87.188 .140 €$ & $3,42 \%$ \\
\hline 9 & GlaxoSmithKline & $77.942 .372 €$ & $3,06 \%$ \\
\hline 10 & Bial & $60.836 .585 €$ & $2,39 \%$ \\
\hline
\end{tabular}

Fonte: IMS Health, origem citada.

mentado por classes terapêuticas ou entidades nosológicas

- A elasticidade procura/preço é mais baixa do que noutros mercados

- O preço dos medicamentos não é suportado na sua totalidade directa e imediatamente pela grande maioria dos utentes, dado os regimes de participação.

O preço de produtos dominados por oligopólios é sempre superior a produtos semelhantes em concorrência aberta. Este problema ainda se torna mais grave quando esse preço é comparticipado e o utilizador/comprador está em situação de fragilidade, ameaçado pela continuidade de situações de doença ou até de morte e não dispõe de informação adequada.

Um outro aspecto distintivo é a relação doente-prescritor, em que o doente se encontra em sofrimento ou necessidade física ou psicológica e procura uma solução para os seus males. O prescritor, normalmente médico, é encarado pelo doente como fonte de bem-estar e quase «deificado». Claro que esta situação é explorada pelas empresas fornecedoras de medicamentos.

São estas as principais causas que determinam a elevada lucratividade do sector a nível global (superior, na maioria dos mercados nacionais, a $20 \%$ das vendas) e a elevadíssima intensidade da promoção.

\section{A INDÚSTRIA FARMACÊUTICA E OS PRESCRITORES}

Uma característica fundamental dos prescritores é a necessidade de informação atempada, em qualidade e quantidade, sobre os medicamentos, apresentada de forma a facilitar a sua utilização no processo de tratamento dos doentes. É claro que a IF tem todo o interesse em facilitar a aquisição de conhecimentos sobre medicamentos pelos médicos, na forma e no modo que lhes seja mais conveniente.

Essencialmente, segundo J. Gropman, da Universidade de Harvard, no seu livro «Como pensam os Médicos», existem vários modos de «aprender» a decidir em Medicina. ${ }^{15} \mathrm{O}$ método mais tradicional é o do «aprendiz» que observa e copia a forma como o mestre age. Raramente, um «aprendiz» tem conhecimento das razões para tomar uma decisão. Ele copia a abordagem dos mais experientes em situações semelhantes. O segundo processo de decisão baseia-se na definição de algoritmos e na técnica da árvore de decisões. Tudo começa com a detecção de um sintoma ou dado laboratorial a que se seguem diversas hipóteses com critérios de aceitação ou rejeição. Esta metodologia é útil para diagnósticos e tratamentos correntes, mas perde utilidade quando os sintomas são múltiplos e confusos e os resultados dos testes inconclusivos, limitando a capacidade de decisão do Médico. Também está na moda basear todas as decisões na observação de sintomas e em dados comprovados estatisticamente. Esta Medicina baseada em factos está a tornar-se um cânone em muitos Hospitais. Quando não existem dados estatísticos comprovados, o sistema emperra.

Não existem estudos que permitam dizer, de forma inequívoca, qual o processo de decisão de um médico no processo de diagnóstico e tratamento. Mas sabemos que a informação desempenha um papel determinante, assim como a experiência e a capacidade de se relacionar com o doente. Muitas vezes, de forma intuitiva, o médico «vê» um determinado quadro clínico. Só depois formula hipóteses que procura confirmar, tendo em conta a informação de que dispõe. Não existe uma 
forma única de fazer um diagnóstico e prescrever um tratamento, mas a informação desempenha um papel central no processo. Não é de modo algum surpreendente que a IF fundamente a informação que transmite em modelos de decisão sobre escolha de tratamentos seguidos pelos médicos.

Um desses modelos, o de Denig e Haaiejer-RusKamp ${ }^{16}$ depois de estudar o processo de decisão de médicos, conclui que o processo de prescrever varia com a cultura médica de cada Escola Médica e é muito influenciado pelas fontes de informação (Quadro III). De acordo com esse modelo, os médicos são influenciados pelas fontes de informação a dois níveis. No primeiro nível os médicos usam o seu conhecimento e experiência profissional para formular um conjunto de informação personalizado, pessoal, ou em termos de terminologia de marketing o «conjunto evocado» (evoked set), para um determinado quadro clínico. O segundo nível diz respeito à tomada de decisão prática de aplicar a sua competência e experiência pessoais e esses conhecimentos para chegar a uma terapêutica individual para cada doente, nomeadamente a escolha de um determinado medicamento. Haaiejer-RusKamp e Denig ${ }^{16}$ indicam que o primeiro conjunto de critérios de escolha provavelmente é adquirido pelo médico enquanto estudante, copiando o comportamento dos seus professores. Estes autores referem também que os procedimentos derivados da aquisição destes comportamentos mudam com pouca frequência.

Uma outra constatação é a de que o médico não é completamente livre na escolha do arsenal terapêutico, sendo limitado pela existência de formulários e pelas directrizes administrativas das instituições e empresas de seguros de que dependem; o preço não é, em geral, um critério de escolha utilizado pelo médico, embora as instituições possam agir no sentido de que ele o considere. Em média, um médico substitui cinco marcas/medicamentos por ano no conjunto do arsenal terapêutico que utiliza. Especialistas e médicos de Clínica Geral apresentam razões diferentes para mudar o seu arsenal terapêutico.

Segundo Jones et al. ${ }^{17}$ os especialistas parecem ter mais em conta a informação de reuniões científicas e a literatura publicada em revistas da sua especialidade, enquanto os clínicos gerais seguem um processo mais difuso com uma clara influência da informação da IF e

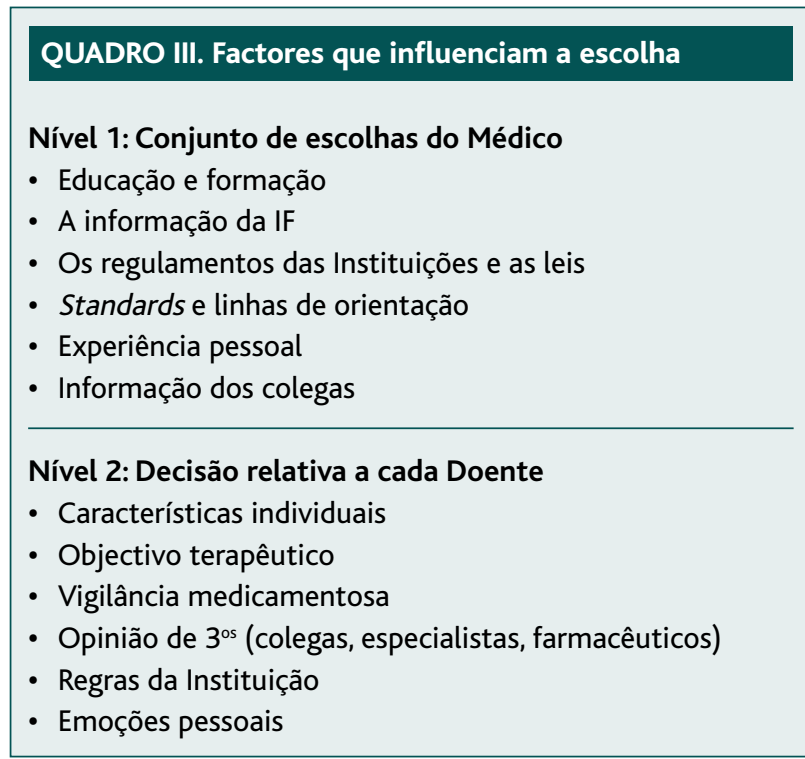

Fonte: Haaiejer-RusKamp e Denig. How does pharmaceutical marketing influence prescribing behaviour, CPB, 2001.

da opinião dos especialistas. Sempre que a população é mais carenciada o médico mostra-se mais relutante em prescrever marcas que não sejam comparticipadas ou que sejam menos comparticipadas e mais caras, especialmente se existirem alternativas de mais baixo preço.

Conhecendo este processo, a IF adapta a informação e o marketing a estas características, procurando fornecer razões para os médicos preferirem uma determinada marca ou aumentar a sua lealdade para com a marca. O resultado é uma elevadíssima percentagem dos proveitos gasta em marketing e uma das mais elevadas rendibilidades de todos os sectores industriais.

A IF tenta explicar este facto com o elevado custo da I\&D e os elevados riscos de insucesso desta actividade. A Associação Americana da Indústria Farmacêutica cita como número de referência que cada molécula nova absorve cerca de meio milhão de dólares em I\&D. Fontes independentes reduzem este número para menos de 120 milhões de euros e alinham argumentos sérios para contrariar a ideia, que a IF quer fazer passar, de que é um negócio arriscado. ${ }^{18}$ De facto, para contrariar a afirmação de que a IF é um negócio de risco devido aos custos da I\&D e à incerteza do resultado desses esforços, basta ter em conta que, nos EUA, a IF foi o sector mais rentável todos os anos desde 1982, de acordo com 
as estimativas anuais da revista Fortune, sendo esta consistência a prova do baixo risco deste negócio. Dado o carácter global da IF, tudo indica que a alta rendibilidade se verifica em todos os mercados, existindo muitos dados sobre este facto no que respeita à U.E. e ao Japão.

\section{AS ACTIVIDADES DE MARKETING DA INDÚSTRIA FARMACÊUTICA}

Analisando as actividades de marketing das diferentes empresas da IF, podemos listar como mais importantes as seguintes:

\section{- Contactos directos com os prescritores}

Aqui não existe qualquer tipo de desproporção entre promotor e receptor, já que os contactos são efectuados com técnicos que têm a capacidade de procurar mais informação e comparar as soluções propostas.

Mas a informação é assimétrica, e alguns efeitos secundários só se revelam, por vezes, no longo prazo, como o «Vioxx», que teve de ser retirado do mercado por «suspeita» de aumentar a frequência dos enfartes do miocárdio.

No entanto, começa-se a procurar limitar o número de contactos que cada empresa pode fazer por ano ou o tipo de contacto que, por exemplo, só se efectivará se um órgão independente concordar que existe nova informação a comunicar ao prescritor, tal como se verifica na Suécia. Cada ano que passa o número de delegados de informação médica (DIMs) tem vindo a aumentar enquanto a sua produtividade tem vindo a diminuir. As principais empresas possuem, na Europa, equipas entre 2.500 e 8.000 DIMs que efectuam cada um cerca de 300 a 700 visitas anuais, em cerca de 180 dias/ano de trabalho de Promoção junto dos Médicos, segundo a IMS Health. ${ }^{6}$

- Uso das Revistas especializadas

A publicação de anúncios em revistas especializadas é limitada por dois tipos de órgãos: o comité científico da revista e a legislação, que só permite que se mencionem nos anúncios vantagens comprovadas por ensaios cientificamente elaborados.

\section{- Contacto directo escrito, via correio, mail, outro}

Está a ser cada vez mais usado o contacto via e-mail. Por outro lado as acções pelo correio estão a diminuir de intensidade. Não existem dados sobre a efec- tividade destas acções, mas tudo indica que têm pouco impacto.

- Recrutamento de prescritores para acções não éticas Este tipo de acções possui uma grande amplitude e abrange desde a organização de viagens ditas científicas até à elaboração de estudos "científicos» enviesados e muito bem pagos, a prescritores líderes de opinião.

\section{- Pesquisa científica orientada}

Algumas empresas, operando no campo da saúde, têm proposto protocolos de investigação aparentemente muito cuidados, mas que escondem, propositadamente, efeitos adversos ou secundários. Isto levou as organizações de médicos e as estruturas de saúde a limitarem fortemente a realização de ensaios clínicos pelos prescritores que nelas exercem a sua actividade.

- Anúncios directos ao consumidor final Uma forte promoção directa ao consumidor, quando legalmente possível, desde que bem dirigida, sustentada de forma continuada e excedendo o limiar mínimo de impacto, tende a aumentar o consumo, só por si, em cerca de $30 \%$ ao ano. Se os produtos já estão maduros, um aumento de $30 \%$ nos anúncios regulares ao público leva ao amento de $40 \%$ nas vendas. Mas os anúncios ao público só são legalmente autorizados no que respeita a medicamentos cuja venda é autorizada sem prescrição.

A crítica que é feita ao anúncio nos grandes órgãos de comunicação social advém do facto de promover a auto-medicação e o uso de drogas em que os efeitos secundários variam de indivíduo para indivíduo. Aliás, a auto-medicação é uma prática que se tem revelado prejudicial para muitos doentes que, em muitos casos, acabam por ter de consultar o Médico.

\section{Em síntese}

- A influência dos DIMs, da publicidade em revistas clínicas e ao público, da organização de reuniões de informação e outros meios promocionais na prescrição está comprovada. Muita dessa influência não é reconhecida pelos prescritores, mas estudos vários provam a sua importância. ${ }^{14,19-21}$

- Os padrões de prescrição são mais influenciados pela actividade dos DIM. Num estudo conduzido sobre a prescrição no serviço de saúde inglês, $100 \%$ das pres- 
crições estavam influenciadas pela acção dos DIM. ${ }^{22}$ Dados dos Estudos de Prescrição da IMS Health nos Países Baixos indicam que 72\% das visitas dos DIM foram consideradas pelo painel de médicos inquirido em 2006, muito úteis, $19 \%$ como tendo uma utilidade média e só $10 \%$ foram consideradas não úteis.

- Os resultados de venda dependem essencialmente da utilidade do produto prescrito, do prestígio da marca promovida, da intensidade do trabalho do DIM (número de visitas efectuado) e da qualidade dessas visitas.

- A influência da publicidade em revistas especializadas também tem importância como reforço da actividade de promoção pessoal.

- A importância do papel dos líderes de opinião está demonstrada, especialmente sobre os médicos mais jovens.

- Por último, a conjugação equilibrada de todos os meios promocionais potencia de forma sinérgica os resultados obtidos.

Assim, qualquer acção promocional da IF conjuga seis tipos distintos de actividades: 1) a actividade dos DIMS, melhorando a sua qualidade e quantidade e usando material promocional de apoio adequado; 2) a comunicação escrita e por mail; 3) a divulgação da posição dos líderes de opinião em artigos de revistas da especialidade ou através de eventos «credíveis» (congressos, simpósios, reuniões, etc.); 4) anúncios em imprensa especializada, muito dirigidos e que têm de complementar outras acções; 5) anúncios de actividades nos jornais e revistas de grande circulação (respeitando as limitações legais); 6) testes de medicamentos (com amostras).

\section{QUAL O PESO DE DADA ACÇÃO?}

Pouco se sabe sobre como as diferentes empresas distribuem por rubricas orçamentais as suas actividades de marketing.

Alguns dados, no entanto, podem ser recolhidos em publicações da IMS Health e noutras fontes, como os departamentos de supervisão das actividades Farmacêuticas na Europa.

De todos os dados consultados o mais significativo, por abranger 28 planos de marketing de diferentes companhias, é o estudo IGZ-2001, conduzido pela Unidade de Supervisão das Actividades de Promoção da Ins- pecção de Saúde dos Países Baixos. ${ }^{23}$ As principais conclusões deste estudo indicam que, nos Países Baixos, o orçamento de marketing das 28 empresas analisadas consome mais de $25 \%$ do total de proveitos anuais, o que significa que quanto maior o volume de vendas maior é a intensidade da promoção. As principais despesas dos orçamentos de marketing referem-se a custos com DIMs, que atingem $68 \%$ do total e promoção ( $20 \%$ do orçamento), restando $32 \%$ para outras actividades.

Excluindo os custos com pessoal, no marketing de produtos específicos com menos de três anos de introdução no mercado, em média, a publicidade pesa $20 \%$, a pesquisa de marketing de produtos já no mercado outros $20 \%$, os cursos e conferências $19 \%$, os envios pelo correio $12 \%$, os eventos promocionais $11 \%$, o «sponsoring» $5 \%$, o apoio a intervenções em conferências de líderes de opinião $3,5 \%$ e outras actividades $9,5 \%$. Estes dados são meramente indicativos e correspondem a médias, podendo verificar-se grandes variações de acordo com o potencial de mercado do produto e a sua posição no ciclo de vida.

A diversidade de actividades promocionais levadas a cabo pela Indústria Farmacêutica visa a sua complementaridade e a obtenção de sinergias. A enorme intensidade dos esforços de marketing é possível dada a rendibilidade do sector e a intenção de recuperar os investimentos o mais rapidamente possível, em produtos com ciclos de vida relativamente curtos.

A sensibilidade da procura ao preço é muito reduzida, dada a dependência do utilizador face ao prescritor (médico) e dados os sistemas de comparticipação. A intensidade da actividade de marketing da IF, em comparação com a informação sobre medicamentos, originada em fontes independentes ou que defendam o interesse público, torna a informação sobre medicamentos fortemente assimétrica (desigual detenção de informação entre os intervenientes num mercado) já que toda a informação sobre os desenvolvimento e testes do produto está na posse da empresa farmacêutica que desenvolve a molécula e é confidencial.

A Industria Farmacêutica controla a informação primária sobre cada nova molécula, os estudos que desenvolve para avaliar a sua aplicabilidade terapêutica e acompanha a evolução do produto depois de entrar no mercado. Para além deste facto, a IF dispõe de estudos 
de mercado muito sofisticados e completos que abrangem os mais importantes mercados mundiais. A IMS Health, principal fonte de dados secundários para a indústria farmacêutica mundial, que opera em cerca de 100 países, possui uma rede global para recolha de dados reais e por amostra que abrange, pelo menos, os médicos, os laboratórios, as farmácias, os armazenistas, os hospitais. Em Portugal, a informação da IMS Health, baseada em painéis, abrange o consumo de especialidades terapêuticas em unidades e valores, por embalagem de produto, distribuída por 15 regiões e emitida com periodicidade mensal sob a forma impressa e/ou digitalizada em bases de dados. O painel de armazenistas abrange mais de $90 \%$ do mercado e está subdividido em 750 micro-regiões ou «Microbricks». O painel de farmácias abrange cerca de 180 farmácias separadas em 5 regiões e o estudo da prescrição médica, com periodicidade trimestral, baseia-se numa amostra de cerca de 550 médicos dividida em seis regiões, compreendendo 15 especialidades médicas, que fornece dados sobre as consultas por diagnóstico e especialidade, principais patologias por sexo e idade, produtos mais prescritos e efeitos desejados; o estudo hospitalar fornece informações sobre o consumo por hospital e serviço numa base trimestral, cobrindo cerca de $40 \%$ do universo dos hospitais centrais e distritais. De uma forma geral, pode-se dizer que a Indústria Farmacêutica influencia fortemente a prescrição dos médicos, em especial a dos Clínicos Gerais.

\section{UM PROBLEMA CONTROVERSO: AS MÁS PRÁTICAS DA IF}

Segundo Hoiman $\mathrm{Chiu}^{24} \mathrm{a}$ análise das tácticas de marketing e investigação da IF revela a sua enorme influência sobre a forma como o médico administra a saúde e como é dirigida a pesquisa Médica. ${ }^{15}$

As más práticas, quando utilizadas, podem ser arrumadas em cinco categorias, de acordo com o prejuízo potencial que podem induzir os médicos a causar nos doentes: 1) promoção enviesada, incompleta ou cientificamente inexacta; 2) anúncios directos aos consumidores e apoio a cadernos de saúde dirigidos ao Público que levam os doentes a pressionarem os médicos para receitarem determinada terapêutica; 3) recrutamento não ético de médicos; 4) ignorar ou ultrapassar intencionalmente conflitos de interesses dos investi- gadores recrutados; 5) manipulação de dados em ensaios clínicos.

Claro que estas práticas, quando usadas, tendem a incentivar a prescrição de medicamentos que não correspondem aos melhores interesses de médicos, doentes e instituições públicas e privadas. Apesar destas práticas não se aplicarem na generalidade, existem e estão referenciadas na literatura médica, nomeadamente em publicações como o Journal of Medical Ethics, o The Lancet, o Journal of General Internal Medicine, o Southern Medical Journal, o American Journal of Obstetrics \& Gynecology.

Algumas destas práticas são também referenciadas, com alguma frequência, nas cartas aos editores destas e de outras revistas, como a Nature, Neuroscience, publicações do National Institute for Health Care Management, etc. Curiosamente, em Portugal é muito rara a denúncia e divulgação de práticas menos éticas, embora muitas delas sejam conhecidas pelos médicos e no meio da saúde.

Pela sua importância ética estes procedimentos deveriam, antes de mais, ser evitados através da adopção de um código de conduta da Indústria Farmacêutica mais rigoroso, e a Apifarma deveria investigar qualquer caso de má prática que chegasse ao seu conhecimento. Também o INFARMED deveria dar maior atenção e fazer análises mais regulares e aprofundadas dos procedimentos da IF, para moralizar radicalmente o sistema.

\section{QUE MELHORIA SERÁ EXEQUÍVEL IMPLANTAR A NÍVEL DOS SISTEMAS DE SAÚDE PARA OPTIMIZAR A UTILIZAÇÃO DE RECURSOS E LIMITAR A PRESSÃO SOBRE OS MÉDICOS?}

Antes de mais, há que reconhecer que existe um conflito de interesses entre a IF, os utentes que pagam ou participam no custo dos medicamentos e os serviços oficiais e privados (por exemplo as companhias de seguros) que comparticipam ou suportam esses custos.

Para limitar o impacto negativo das actividade da IF poder-se-á desenvolver um distinto tipo de acções:

- Aumentar, a curto prazo, a sensibilidade ao preço por parte dos prescritores.

- Para atingir este objectivo é essencial que a prescrição médica se faça por DCI e que as entidades públicas divulguem e garantam a equivalência terapêutica e química e a qualidade entre os diferen- 
tes produtos no Mercado com a mesma aplicação terapêutica, reforçando a comunicação de dados relevantes para a prescrição através de informação de alta qualidade, para diminuir a assimetria de informação agora prevalecente.

- Um cuidado a ter é o de limitar a influência directa da IF sobre as entidades que elaboram esta informação, de forma a mantê-la de facto independente.

- Reforçar, no Sistema Nacional de Saúde, a participação nos medicamentos quimicamente equivalentes de mais baixo custo e diminuir a participação nos de mais alto custo.

- Aumentar a importância da análise custo/benefício na decisão de quais os Medicamentos comparticipados e qual a taxa de participação, de tal forma que as diferenças nas taxas de comparticipação reflictam a diferença de qualidade terapêutica. Como é evidente, estas diferenças não poderiam existir entre produtos substitutos com acção bioquímica muito semelhante (close substitutes).

- Incentivar a utilização pelos Médicos de programas de prescrição informatizados que facilitem a escolha das melhores opções de prescrição, em termos de custo benefício e eficiência terapêutica.

- Criar incentivos financeiros significativos para os Médicos que receitam medicamentos participados de mais baixo custo.

- Incentivar a adopção de um Código de Conduta da Associação da Indústria Farmacêutica que auto-regule a sua actividade de marketing.

- Regulamentar, gradualmente, a actividade dos contactos IF-Prescritores e exercer controlo efectivo sobre a informação contida no material publicitário distribuído e sobre a transmitida oralmente.

- Proceder a estudos comparativos dos preços e custos para os Utentes em diferentes países europeus e publicitar as conclusões de forma a desmistificar as mensagens erróneas transmitidas por muitas empresas da IF, nomeadamente quanto à qualidade $\mathrm{e}$ origem das matérias-primas que utilizam e quanto aos preços.

- Considerar o papel real das farmácias no aconselhamento do fármaco a usar pelo doente e permitir a substituição farmacêutica do receituário por produto equivalente, incentivando o Utente a escolher um medicamento quimicamente igual de mais baixo custo, quando do aviamento na Farmácia, depois de informado das diferentes opções.

A adopção destas e doutras medidas complementares iria aumentar a independência da decisão dos médicos no seu receituário e induzir uma diminuição das despesas em marketing da IF, permitindo uma descida gradual dos preços. Dada a necessidade de resolver o agudo conflito de interesses entre IF e Estado que comparticipa ou paga os medicamentos e os utentes e cidadãos que pagam os seus impostos, é urgente que se inicie um programa estruturado que elimine a prevalência actual dos interesses da indústria farmacêutica.

\section{ENDEREÇO PARA CORRESPONDÊNCIA}

Luís dos Santos Ferreira

E-mail: ecosferreira@netcabo.pt

\section{REFERÊNCIAS BIBLIOGRÁFICAS}

1. World Health Organization Resolution WHA40.43 - Technical Cooperation. Geneva: World Health Organization; 1977.

2. Proceedings of the Symposium on World Health Day. Geneva: WHO; 2007.

3. OECD Health Division (2006) OECD Health Data 2006, October 06. Disponível em: http://www.oecd.org/dataoecd/20/51/37622205.xls [acedido em 26/10/2008].

4. Bentes M, Dias CM, Sakellarides C. Health care systems in transition: Portugal. Copenhagen:The European Observatory on Health Care Systems; 2003.

5. Dec.-Lei 71/91, de 8 de Fevereiro.

6. IMS Health. Disponível em: http://www.imshealth.com [acedido em 30/10(2008]

7. Aguiar AH. Política do Medicamento: Guia de Consulta. Lisboa: AJE; 2004.

8. Frank RG, Seiguer E. Generic drug competition in the US. Business Briefings Pharmagenetics 2003. Disponível em: http://www.touchbriefings.com/download.cfm?filelD=281 [acedido em 28/10/2008].

9. Diário de Notícias, 31/05/2006. Disponível em: http://dn.sapo.pt/ 2006/12/15/sociedade/farmacos_vendidos_a_preco_excessivo.html [acedido em 30/10/2008].

10. Diário Económico, 30 de Maio de 2007.

11. Jacobezone S. Pharmaceutical policies in OCDE countries: reconciling social and industrial goals, labour market and social policy. Ocasional paper, $\mathrm{n}^{\circ}$ 40. Paris: Organization economic co-operation and development; 2000.

12. Ballence R, Forstner $H$, Sawyer WC. An empirical examination of the role of vertical product differentiation in North-South trade. Weltwirtsch Arch 1992; 128 (2): 330-8.

13. Scherer FM, Ross D. Industrial market structure and economic performance. 3rd ed. Boston, MA: Houghton Mifflin Company; 1990.

14. Laat $E$, Windmeijer F, Douven R. How does pharmaceutical marketing influence doctors' prescribing behaviour? Den Hagen: CPB Nether- 
lands' Bureau for Economic Policy Analysis; 2002.

15. Gropman J. Como pensam os médicos. Lisboa: Casa das Letras; 2008

16. Popa C. DU90 for the assessment of drug prescribing in primary care [Dissertação de mestrado]. Göteborg: Nordiska Högskolan för Folkälsovetenskap; 2005. Disponível em: http://www.nhv.se/upload/ dokument/forskning/Publikationer/MPH/MPH\%202005-18\%20CPopa.pdf [acedido em 28/10/2008].

17. Jones MI, Greenfield SM, Bradley CP. Prescribing new drugs: qualitative study of influences on consultants and general practitioners. BMJ 2001 Aug 18; 323 (7309): 378-85.

18. Public Citizen's Congress Watch. Rx R\&D Myths: the case against the drug industry's R\&D "Scare Card". Public Citizen, Washington, 2001. Disponível em: http://www.citizen.org/congress/campaign/special_interest/articles.cfm?ID=6538 [acedido em 28/10/2008].

19. Wazana A. Physicians and the pharmaceutical industry: is a gift ever just a gift? JAMA 2000 Jan 19; 283 (3): 373-80.
20. Szalkai Z. Relationship marketing in the pharmaceutical industry: analysis of the Hungarian case. Periodica Politecnica 2004, 12 (2): 177-88.

21. Blumenthal D. Doctors and drug companies. N Engl J Med 2004 Oct 28; 351 18: 1885-90.

22. GP Prescribing Behaviour 2006: Report into the findings of a survey of General Practitioners in England: prepared for the National Audit Office. Disponível em: www.nao.org.uk/publications/nao_reports/0607/GP_survey_final_report_from_Doctors_Net.pdf [acedido em 30/10/2008].

23. Dutch Advertising Supervision Unit of the Health Care Inspection. Study IGZ 2001.

24. Chiu H. Selling drugs: marketing strategies in the pharmaceutical industry and their effect on healthcare and research. Explorations: UC Davis Undergraduate Research Journal 2005; 8: 89-94. Disponível em: http://undergraduatestudies.ucdavis.edu/explorations/2005/explorations.pdf [acedido em 01/10/2008].

\section{ABSTRACT}

The present article introduces a very synthetical insight into Portugal's healthcare situation and its position with regards to countries that have a similar degree of development. It describes the enduring inequities and in particular the fact that health-related costs have a significant importance with regards to the GDP and the fact that such costs are disproportionate to their amplitude and quality. The article also specifies the process of healthcare delivery and the crucial role played by the doctor and the drugs.

Following a brief depiction of the pharmaceutical industry, we analyze the relation between pharmaceutical marketing and the doctor as prescriber. Two aspects are highlighted in the analysis: the importance of information in the medical prescription and the profound asymmetry between information conveyed by the pharmaceutical industry and independent information. This asymmetry throws into relief the relation between the intensity of the prescription of a given brand and the intensity of the information that the pharmaceutical industry sends out to the prescriber.

In addition, the article analyses the means used by the industry to promote its products and their significance in the companies' spending structures; it also points out inappropriate practices and measures that strive to limit its negative effects.

Keywords: Drugs; Marketing; Physicians; Health Care Costs. 